\title{
Collision and composition tumors: rare conditions to remember in differential diagnosis of adrenal glands lesions
}

\author{
Giuseppe Cicero, Alfredo Blandino, Stefano Pergolizzi, Giorgio Ascenti, Silvio Mazziotti \\ Section of Radiological Sciences, Department of Biomedical Sciences and Morphological and Functional Imaging, University of Messina, Messina, \\ Italy
}

Correspondence to: Giuseppe Cicero, MD. Department of Biomedical Sciences, and Morphological and Functional Imaging, University of Messina, Via Consolare Valeria, 1, 98100 Messina, Italy. Email: gcicero87@gmail.com.

Submitted Apr 11, 2019. Accepted for publication May 08, 2019.

doi: 10.21037/qims.2019.05.11

View this article at: http://dx.doi.org/10.21037/qims.2019.05.11

We have read with interest a recently published article entitled "CT and MRI of adrenal gland pathologies" by Wang et al. (1). The article provides a wide overview focused on the typical computed tomography (CT) and magnetic resonance imaging (MRI) features related to several pathological conditions that can involve the adrenal glands, with particular attention to the ones associated to hormones secretion and endocrine syndromes.

Considering the huge variety of both the benign and the malignant lesions and the number of radiological findings, the differential diagnosis can often be challenging. Furthermore, the simultaneous occurrence of different lesions within the same adrenal gland can considerably increase the difficulty of a proper differential diagnosis. Generally speaking, the term "collision tumors" is referred to the presence of at least two contiguous tumors with different histological features (2). Some authors further distinguish "collision tumors", characterized by the coexistence of two different primary neoplasms, from "collision phenomenons", related to the simultaneous occurrence of a primary neoplasm and a metastasis or to the concurrent presence of two different metastasis arising in the same site (3). As regards the adrenal glands, when adjacent but independent lesions are identified, three pathological scenarios can be defined: adrenal collision tumors, if the admixture at the interface between the two lesions is not significant; composition (or composite) tumors, when the "confluent zone" is significant; combination tumor, if the different pathologic components originate from a common stem cell $(2,4-6)$.
However, the distinction between composition and combination tumors is generally not considered anymore (4). Currently, although only few data exist about their epidemiology, these lesions are generally considered of rare occurrence $(<2 \%)(4,6)$. Different histopathological hypotheses have also been pushed forward, such as the coincident onset of different tumors, or the presence of a pre-existing tumor determining tissue alterations that promote the development of a second neoplasm $(4,6)$. CTscan is usually the first imaging modality employed for detection and characterization of adrenal lesions. The CT features and enhancement patterns are usually accurate enough in discriminating between benign and malignant behaviors. Owing to its greater detail on soft tissues, MRI can be performed as "problem solving" when CT findings are not conclusive. PET/CT with ${ }^{18} \mathrm{~F}$-fluorodeoxyglucose (FDG) uptake is also able to recognize malignant components included in the adrenal lesions $(5,6)$. If doubts persist, percutaneous biopsy can also be performed, although the sampling requires a very high level of accuracy since the lesions are adjacent and usually of small size (6).

We came across a 62 -year-old male affected by colon cancer. The abdominal MRI led to detect a plenty of bulky metastasis within the liver and a large right adrenal gland lesion on chemical-shift images (CSI), furthermore characterized by a signal drop in the out-of-phase image, suggestive for adenoma. However, on the medial edge of the lesion, a small different lesion was also detectable on the axial T2-w Turbo Spin-Echo (TSE) sequence, whose intensity signal was similar to that of the hepatic 

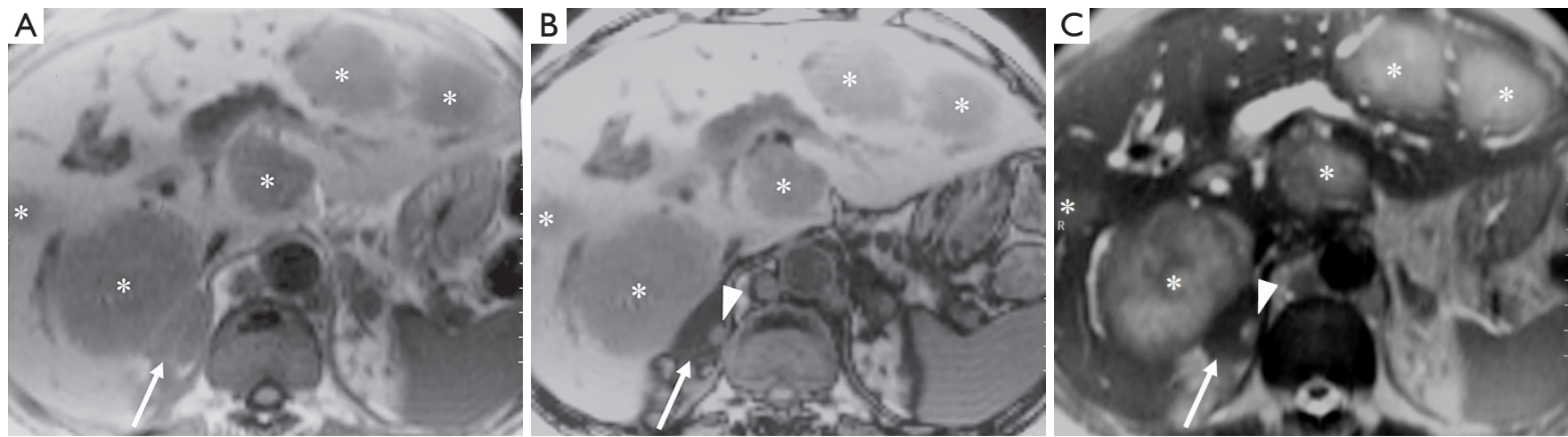

Figure 1 Axial T1-w FFE in-phase image (A) shows several hepatic metastasis (asterisks) and a right adrenal gland lesion (arrow). Axial T1-w FFE out-phase scan (B) demonstrates the signal dropout of the adrenal gland, suggestive for adenoma (arrow), in which is detectable a small hyperintense lesion (arrowhead). On the axial T2-w TSE sequence (C) the signal intensity of the small adrenal lesion (arrowhead) is similar to that of the hepatic lesions, and thus consistent with the metastatic nature.

lesions and thus consistent with the metastatic nature (Figure 1). Adrenal collision and composition tumors should be suspected every time a heterogeneous adrenal gland mass is identified or when a sudden change in lesion appearance or size is detected $(5,7)$. However, degeneration phenomena (i.e., hemorrhage and/or fibrosis) may also arise within a lesion and they have to be taken into account in the differential diagnosis evaluation (6). Radiologists and physicians should be well aware about all these contingencies in order to avoid misdiagnosis. Therefore, the aim of this paper is to supplement the literature data with our experience in this field.

\section{Acknowledgments}

None.

\section{Footnote}

Conflicts of Interest: The authors have no conflicts of interest to declare.

\section{References}

1. Wang F, Liu J, Zhang R, Bai Y, Li C, Li B, Liu H, Zhang T. $\mathrm{CT}$ and MRI of adrenal gland pathologies. Quant Imaging Med Surg 2018;8:853-75.
2. Schieda N, Siegelman ES. Update on CT and MRI of Adrenal Nodules. AJR Am J Roentgenol 2017;208:1206-17.

3. Gravante G, Ong SL, Cameron IC, Richards C, Metcalfe MS, Dennison AR, Lloyd DM. Two primary tumours metastasizing to the liver in a collision phenomenon. ANZ J Surg 2010;80:368-9.

4. Schwartz LH, Macari M, Huvos AG, Panicek DM. Collision tumors of the adrenal gland: demonstration and characterization at MR imaging. Radiology 1996;201:757-60.

5. Shin YR, Kim KA. Imaging Features of Various Adrenal Neoplastic Lesions on Radiologic and Nuclear Medicine Imaging. AJR Am J Roentgenol 2015;205:554-63.

6. Katabathina VS, Flaherty E, Kaza R, Ojili V, Chintapalli $\mathrm{KN}$, Prasad SR. Adrenal collision tumors and their mimics: multimodality imaging findings. Cancer Imaging 2013;13:602-10.

7. Johnson PT, Horton KM, Fishman EK. Adrenal mass imaging with multidetector CT: pathologic conditions, pearls, and pitfalls. Radiographics 2009;29:1333-51.

Cite this article as: Cicero G, Blandino A, Pergolizzi S, Ascenti G, Mazziotti S. Collision and composition tumors: rare conditions to remember in differential diagnosis of adrenal glands lesions. Quant Imaging Med Surg 2019;9(11):1902-1903. doi: 10.21037/qims.2019.05.11 